\title{
Bioinformatics identification of dysregulated microRNAs in triple negative breast cancer based on microRNA expression profiling
}

\author{
JUNQING CHEN $^{1 *}$, ZHANHONG CHEN ${ }^{1 *}$, JIAN HUANG $^{1}$, FENG CHEN $^{2}$, \\ WEIWU YE ${ }^{1}$, GUOJUN DING ${ }^{3}$ and XIAOJIA WANG ${ }^{1}$ \\ Departments of ${ }^{1}$ Medical Oncology, ${ }^{2}$ Breast Surgical Oncology and ${ }^{3}$ Radiology, \\ Zhejiang Cancer Hospital, Hangzhou, Zhejiang 310022, P.R. China
}

Received January 11, 2017; Accepted November 7, 2017

DOI: $10.3892 / \mathrm{ol} .2017 .7707$

\begin{abstract}
Triple negative breast cancer (TNBC) accounts for approximately $15-20 \%$ of all breast cancer cases and is usually more aggressive with a poorer clinical outcome compared with other breast cancer subtypes. Evidence of the involvement of microRNAs (miRNAs) in cancer has provided an opportunity for the development of novel effective therapeutic targets in TNBC. In the present study, the miRNA expression profiles of the human breast cancer cell line, MDA-MB-231, and MCF-7 cells, was evaluated by using miRNA microarray analysis. A total of 107 differentially expressed miRNAs (57 upregulated and 50 downregulated) were identified in MDA-MB-231 cells compared with MCF-7 cells. Five prominently dysregulated miRNAs (miR-200c-3p, miR-221-3p, miR-222-3p, miR-192-5p and miR-146a) were further confirmed by reverse transcription-quantitative polymerase chain reaction. In addition, gene ontology analysis and pathway enrichment analysis revealed that the dysregulated miRNAs and predicted targets were found to be involved in the mitogen-activated protein kinase, Wnt, and transforming growth factor- $\beta$ signaling pathways, which were known to contribute to TNBC progression and metastasis. Finally, miRNA gene network analyses suggested that miR-200c may serve as a crucial miRNA in breast cancer. Taken together, these findings may provide a comprehensive view of the function of aberrant miRNAs involved in TNBC, and dysregulated miRNAs hold promise as potential biomarkers and therapeutic targets for patients with TNBC.
\end{abstract}

Correspondence to: Professor Xiaojia Wang, Department of Medical Oncology, Zhejiang Cancer Hospital, 38 Guangji Road, Hangzhou, Zhejiang 310022, P.R. China

E-mail:wangxiaojia03@sina.com

"Contributed equally

Key words: triple negative breast cancer, miRNA, bioinformatics, biomarker, therapeutic target

\section{Introduction}

Breast cancer is the most common malignant tumor among women and is a leading cause of cancer-associated death in women worldwide. Based on gene expression patterns, breast cancer was divided into five distinct molecular subtypes including luminal $\mathrm{A}$, luminal $\mathrm{B}$, receptor tyrosine-protein kinase erbB-2 (HER2)-enriched, basal-like and normal-like subtype (1). Triple negative breast cancer (TNBC) is defined as the absence of estrogen receptor (ER), progesterone receptor (PR) and HER2 expression, accounting for approximately $15-20 \%$ of all breast cancer patients (2). The majority of TNBC patients (up to 70\%) overlap with the basal-like gene expression subtype (3). Compared with other breast cancer subtypes, TNBC lacks clinically efficient targeted therapies and is usually more aggressive with higher rates of distant metastasis and poorer overall survival (2). Therefore, it is imperative to better elucidate the underlying mechanism of TNBC and identify novel targets for effective therapies.

microRNAs (miRNAs) are a class of small non-coding RNAs that are 19-25 nucleotides in size, which regulate gene expression primarily through mRNA degradation or translational repression. It has been estimated that approximately over one third of human protein-coding genes appeared to be conserved miRNA targets, which suggests that miRNAs may serve an important role in gene expression (4). miRNAs are found to be critically involved in various cancer-associated cell processes, including proliferation, differentiation, cell-cycle control, apoptosis, invasion and metastasis $(5,6)$. miR-9 was demonstrated to be associated with epithelial-mesenchymal transition, aggressive phenotype and poor prognosis in breast cancer, suggesting that miR-9 may serve as a promising biomarker for breast cancer progression (7). It was reported that miR-145 expression was downregulated in MDA-MB-231 cells and overexpression of miR-145 inhibited tumor invasion by targeting ARF6, a known regulator of breast tumor cell invasion (8). However, the miRNA expression profile in TNBC and the role of miRNAs in modulating the pathways of TNBC remain largely unexplored.

In the present study, 107 aberrant miRNAs were identified in human MDA-MB-231 breast cancer cells, compared with MCF-7 cells by means of miRNA microarray analysis. Five prominently dysregulated miRNAs (miR-200c-3p, 
miR-221-3p, miR-222-3p, miR-192-5p and miR-146a) were further validated by reverse transcription-quantitative polymerase chain reaction (RT-qPCR). In addition, bioinformatic analysis including gene ontology analysis and pathway enrichment analysis, were performed in an attempt to reveal the potential roles of these dysregulated miRNAs and associated signaling pathways involved in development and progression of TNBC.

\section{Materials and methods}

Cell culture. Human breast cancer cell lines MDA-MB-231 (ER, PR and HER2 negative) and MCF-7 (ER, PR positive and HER 2 negative) were obtained from the Type Culture Collection of Chinese Academy of Sciences (Shanghai, China). MDA-MB-231 cells were maintained in Leibovitz's L-15 medium (Gibco; Thermo Fisher Scientific, Inc., Waltham, MA, USA) supplemented with $10 \%$ fetal bovine serum (Hyclone; GE Healthcare, Chicago, IL, USA), $100 \mathrm{U} / \mathrm{ml}$ penicillin and $100 \mu \mathrm{g} / \mathrm{ml}$ streptomycin (Invitrogen; Thermo Fisher Scientific, Inc.). MCF-7 cells were cultured in RPMI-1640 medium (Gibco; Thermo Fisher Scientific, Inc.) supplemented with $10 \%$ fetal bovine serum (Hyclone; GE Healthcare), $100 \mathrm{U} / \mathrm{ml}$ penicillin and $100 \mu \mathrm{g} / \mathrm{ml}$ streptomycin (Invitrogen; Thermo Fisher Scientific, Inc.). Cell lines were cultured and maintained at $37^{\circ} \mathrm{C}$ in a humidified atmosphere containing $5 \%$ carbon dioxide.

miRNA microarray profiling. miRNA microarray analysis including separation, quality control, labeling, hybridization and scanning was performed by LC Sciences (Houston, TX, USA). The array contained 1090 human mature miRNA probes for all human mature miRNAs based on Sanger miRase Release 15.0 (The Wellcome Trust Sanger Institute, Hinton, UK). Hybridization was performed on a $\mu$ Paraflo microfluidic chip using a micro-circulation pump (Atactic Technologies, Inc., Houston, TX, USA). Images were collected using a laser scanner (GenePix 4000B; Molecular Devices, LLC, Sunnyvale, CA, USA) and digitized using Array-Pro image analysis software (Media Cybernetics, Inc., Rockville, MD, USA). The data were analyzed by first subtracting the background and then normalizing the signals using a LOWESS filter (Locally-weighted Regression). Student's t-test analysis was conducted to identify differentially expressed miRNAs between MDA-MB-231 and MCF-7 cells. The false discovery rate (FDR) was $\mathrm{P}<0.05$ and $\mathrm{P}<0.01$ and served as the cut-off criteria. The data were $\log 2$ transformed and median centered by Cluster 3.0 software (Informer Technologies Inc., Los Angeles, CA, USA) and then further analyzed with hierarchical clustering with average linkage.

$R T$-qPCR for miRNA expression. Total RNA from cultured cells was extracted using TRIzol reagent (Invitrogen; Thermo Fisher Scientific, Inc.) according to the manufacturer's protocol. The concentration and quality of RNA was determined using the Nanodrop 1000 spectrophotometer (Thermo Fisher Scientific, Inc.). RNA (1 $\mu \mathrm{g})$ was reverse transcribed using Ncode ${ }^{\mathrm{TM}}$ miRNA First-Strand cDNA Synthesis kit (Invitrogen; Thermo Fisher Scientific, Inc.) according to the manufacturer's protocol. The forward primers used for amplification were as follows: miR-200c-3p: 5'-UAAUAC UGCCGGGUAAUGAUGGA-3'; miR-221-3p: 5'-GCTACA TTGTCTGCTGGGTTTC-3'; miR-222-3p: 5'-GCTACATCT GGCTACTGGGT-3'; miR-192-5p: 5'-GCTGACCTATGA ATTGACAGCC-3'; miR-146a: 5'-CAGTGCGTGTCGTGG AGT-3', and U6 snRNA: 5'-GCTTCGGCAGCACATATA CTAAAAT-3'. The universal reverse primer was 5'-GAGACT GCGGATGTATAGAACTTGA-3'. Quantitative PCR was performed using SYBR ${ }^{\circledast}$ Green Realtime PCR Master mix kits (Toyobo Life Science, Osaka, Japan) on a Bio-Rad CFX384 PCR instrument (Bio-Rad Laboratories, Inc., Hercules, CA, USA) according to the manufacturer's protocol. The PCR thermocycling conditions were as follows: Initial denaturation at $95^{\circ} \mathrm{C}$ for $10 \mathrm{~min}$ followed by 40 cycles of $95^{\circ} \mathrm{C}$ for $15 \mathrm{sec}$ and $63^{\circ} \mathrm{C}$ for $25 \mathrm{sec}$. U6 snRNA was used as an internal control. Each sample was run in triplicate. The level of miRNA expression was measured using the $2^{-\Delta \Delta \mathrm{Cq}}$ method (9). The results are presented as fold-change of each miRNA in the MDA-MB-231 cells relative to the MCF-7 cells.

Target prediction and bioinformatic analysis. Targets of miRNAs were predicted by using online miRNA target prediction algorithms: TargetScan, PicTar4, and miRanda (www.targetscan.org, http://pictar.mdc-berlin.de and www. microrna.org, respectively), which are commonly used to predict the targets of miRNAs. The intersection of miRNA target genes was selected for further analysis. Gene ontology (GO) analysis was performed at three levels: Molecular function, biological process and cellular component. Pathway analysis was performed using the Kyoto Encyclopedia of Genes and Genomes database (KEGG) (www.genome. jp/kegg). Functional enrichment and pathway enrichment analyses were performed by using the Database for Annotation, Visualization and Integrated Discovery (DAVID software; http://david-d.ncifcrf.gov) (10). P-value was adjusted by the method of Benjamini-Hochberg to control the false discovery rate (11). Enriched GO terms and KEGG pathways were identified as significant with $\mathrm{P}<0.05$. Cytoscape 2.8.3 (www.cytoscape.org) software was applied to construct the possible functional network of the selected miRNAs and targets (12).

Statistical analysis. Statistical analysis was performed by using the SPSS version 16.0 statistical package (SPSS, Inc., Chicago, IL, USA) using the Student's t-test. $\mathrm{P}<0.05$ was considered to indicate a statistically significant difference.

\section{Results}

Identification of dysregulated miRNAs in MDA-MB-231 cells. To investigate the miRNA profiles in triple negative breast cancer, human breast cancer cell lines MDA-MB-231 (ER, PR and HER2 negative) and MCF-7 (ER, PR positive and HER2 negative) were selected. The miRNA expression profiles of MDA-MB-231 and MCF-7 cells were evaluated by using miRNA microarray analysis. The cluster analysis revealed that MDA-MB-231 cells were characterized by significant alterations in miRNA expression compared with MCF-7 cells. As shown in Fig. 1, 107 miRNAs were identified that were differentially expressed in MDA-MB-231 cells compared with 


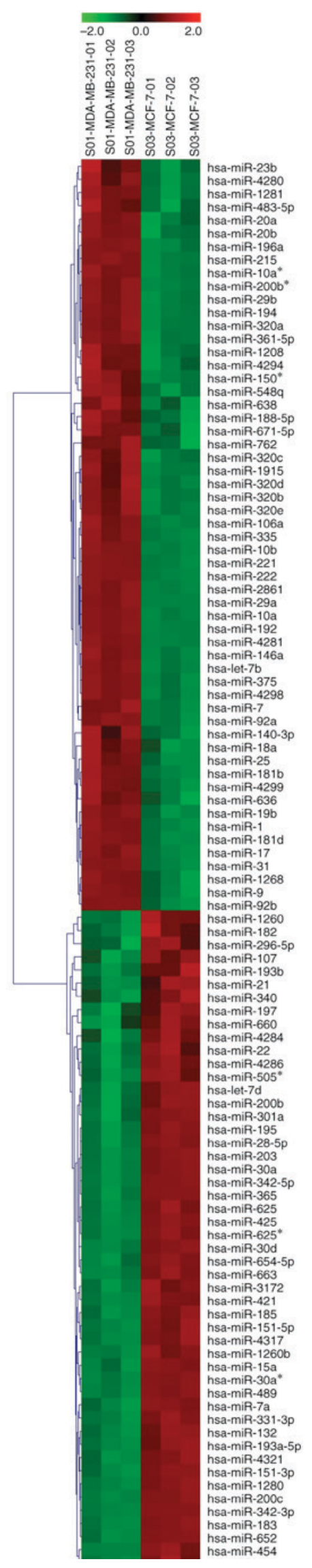

Figure 1. Alterations in miRNA expression profiles between MDA-MB-231 cells and MCF-7 cells by miRNA microarray analysis. A total of 107 miRNAs (57 miRNAs upregulated, 50 miRNAs downregulated) were differentially expressed in MDA-MB-231 cells compared with MCF-7 cells. Red and green indicate upregulated and downregulated miRNAs, respectively. miRNA, microRNA.

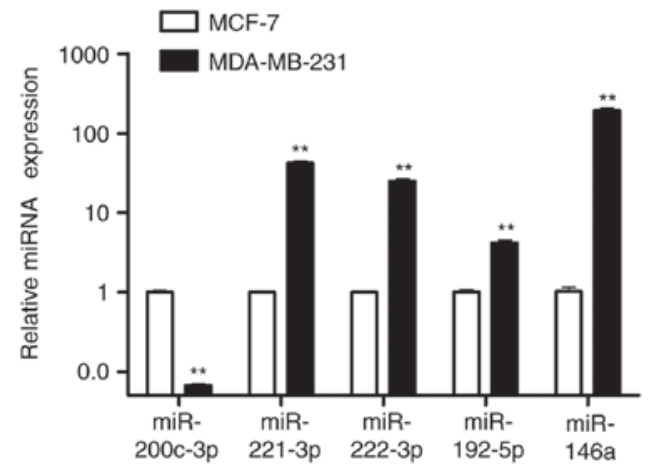

Figure 2. Validation of dysregulated miRNA expression (miR-200c-3p, miR-221-3p, miR-222-3p, miR-192-5p and miR-146a) in MDA-MB-231 cells and MCF-7 cells by reverse transcription-quantitative polymerase chain reaction. miR-200c-3p expression was downregulated and the expression levels of miR-221-3p, miR-222-3p, miR-192-5p and miR-146a were upregulated in MDA-MB-231 cells compared with MCF-7 cells. ${ }^{* *} \mathrm{P}<0.01$ vs. MCF-7. miRNA, microRNA; miR, microRNA.

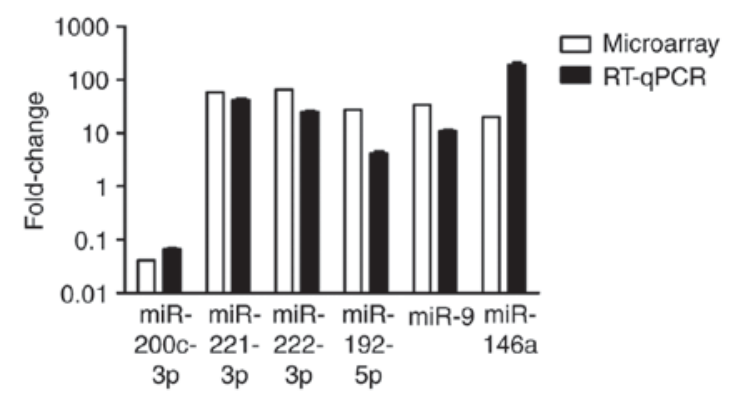

Figure 3. Comparison of miRNA fold-changes by miRNA microarray and reverse transcription-quantitative polymerase chain reaction for miR-200c-3p, miR-221-3p, miR-222-3p, miR-192-5p and miR-146a. The fold-changes were determined in MDA-MB-231 cells compared with MCF-7 cells. miRNA, microRNA; miR, microRNA.

MCF-7 cells $(F D R<0.05 ; \mathrm{P}<0.01)$. Among the 107 miRNAs, 57 miRNAs were upregulated, and 50 miRNAs were downregulated in MDA-MB-231 cells.

Validation of miRNA expression by RT-qPCR. Of the 107 differentially expressed miRNAs obtained from the microarray analysis, the top 5 dysregulated miRNAs (miR-200c-3p, miR-221-3p, miR-222-3p, miR-192-5p and miR-146a) were further verified by RT-qPCR. It was found that there was a downregulation in miR-200c-3p expression and a significant upregulation of miR-221-3p, miR-222-3p, miR-192-5p and miR-146a expression in MDA-MB-231 cells compared with MCF-7 cells (Fig. 2). Fig. 3 shows a comparison of microarray and RT-qPCR results for the five miRNAs. The results of RT-qPCR consistently confirmed the results obtained by microarray analysis.

$G O$ analysis. To elucidate functions of dysregulated miRNAs in TNBC, potential targets of five prominently dysregulated miRNAs validated by RT-qPCR were predicted by miRNA target prediction algorithms. A total of 597 target genes were identified for further analysis. Functional enrichment analysis was performed using DAVID. A total of 955, 84 and $60 \mathrm{GO}$ items were obtained, respectively, in the ontologies of biological 


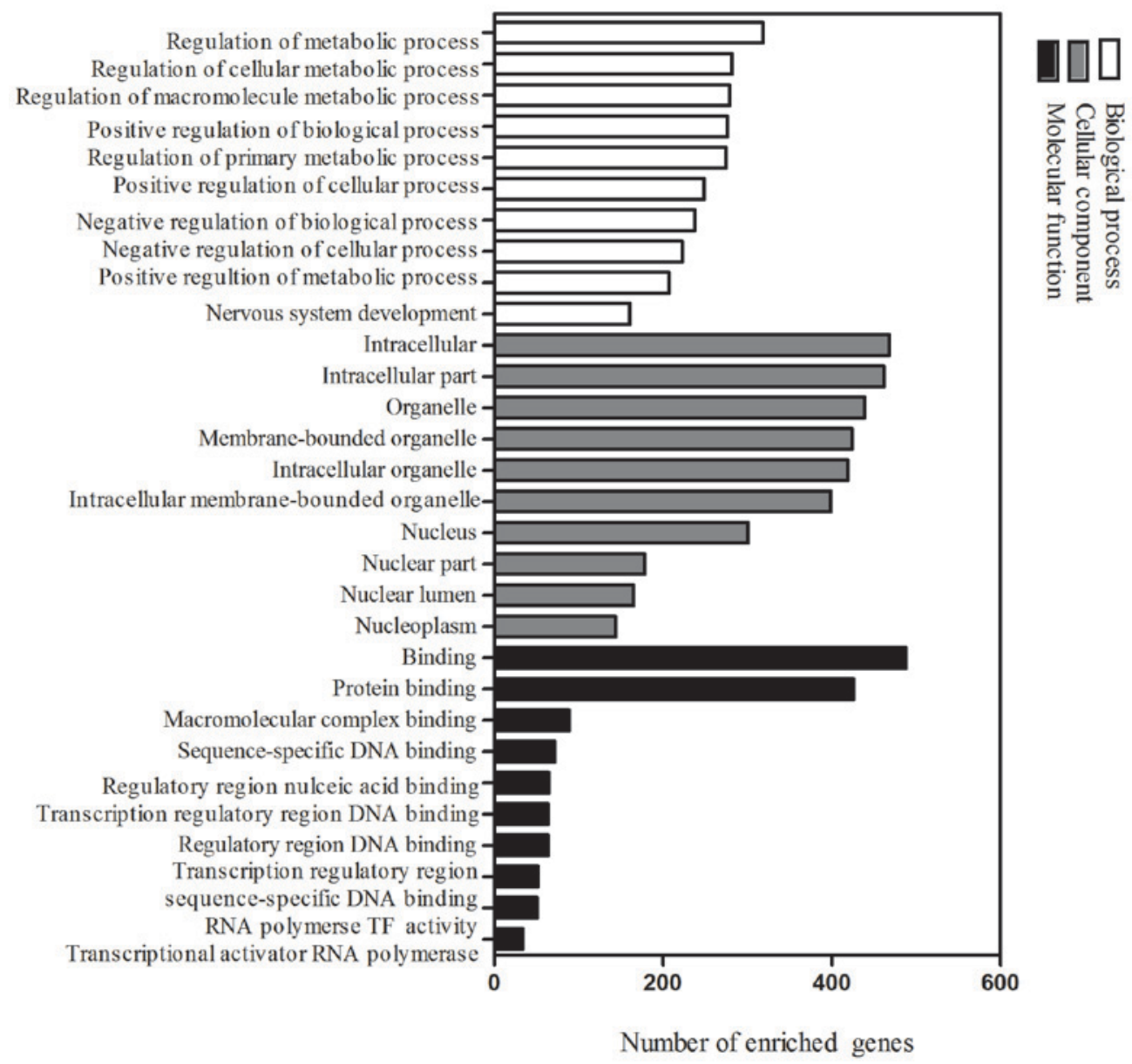

Figure 4. GO analysis of potential target genes regulated by key miRNAs. The 10 most significant items for each ontology including molecular function, biological process and cellular component are presented. GO, Gene Ontology; miRNA, microRNA.

process, cellular component and molecular function $(\mathrm{P}<0.05)$. The 10 most significantly associated terms for each ontology are demonstrated in Fig. 4. It was found that predicted targets of selected miRNAs were involved in important processes such as regulation of metabolic process, transcription factor activity, DNA binding and protein binding.

KEGG pathway analysis and network analysis. KEGG pathway enrichment analysis was performed by using DAVID. There were 53 pathways obtained and 35 of them were statistically significant $(\mathrm{P}<0.05)$. Of these, important pathways involved in tumorigenesis and metastasis were enriched, including the MAPK signaling pathway, Wnt signaling pathway, TGF- $\beta$ signaling pathway, pathways in cancer, cyclic adenosine monophosphate (cAMP) signaling pathway, miRNAs in cancer, transcriptional dysregulation in cancer, AMP-activated protein kinase (AMPK) signaling pathway, ubiquitin mediated proteolysis, cell cycle, adherens junction and gap junction. The top 20 highly enriched KEGG pathways are presented in Fig. 5. To further elucidate associations between selected dysregulated miRNAs and potential target genes, miRNA-gene network analysis was constructed by Cytoscape software (Fig. 6). As shown in the network, miR-200c may regulate several hundreds of target genes, suggesting that miR-200c may serve important roles in breast cancer.

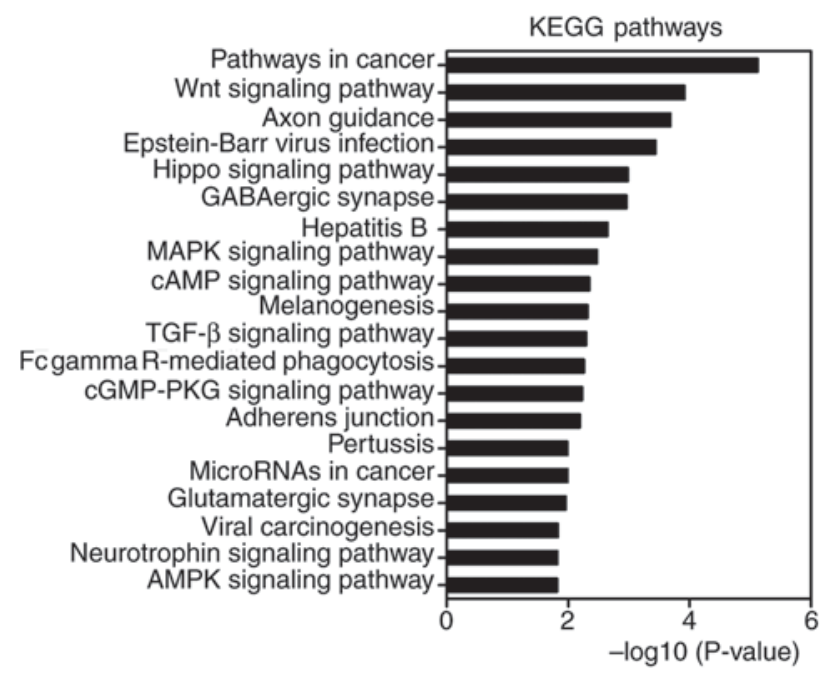

Figure 5. KEGG pathway enrichment analysis performed by using DAVID. The top 20 highly enriched KEGG pathways are presented. KEGG, Kyoto Encyclopedia of Genes and Genomes database; DAVID, Database for Annotation, Visualization and Integrated Discovery.

\section{Discussion}

Breast cancer is considered a complex and heterogeneous disease with distinct molecular subtypes and therapeutic 


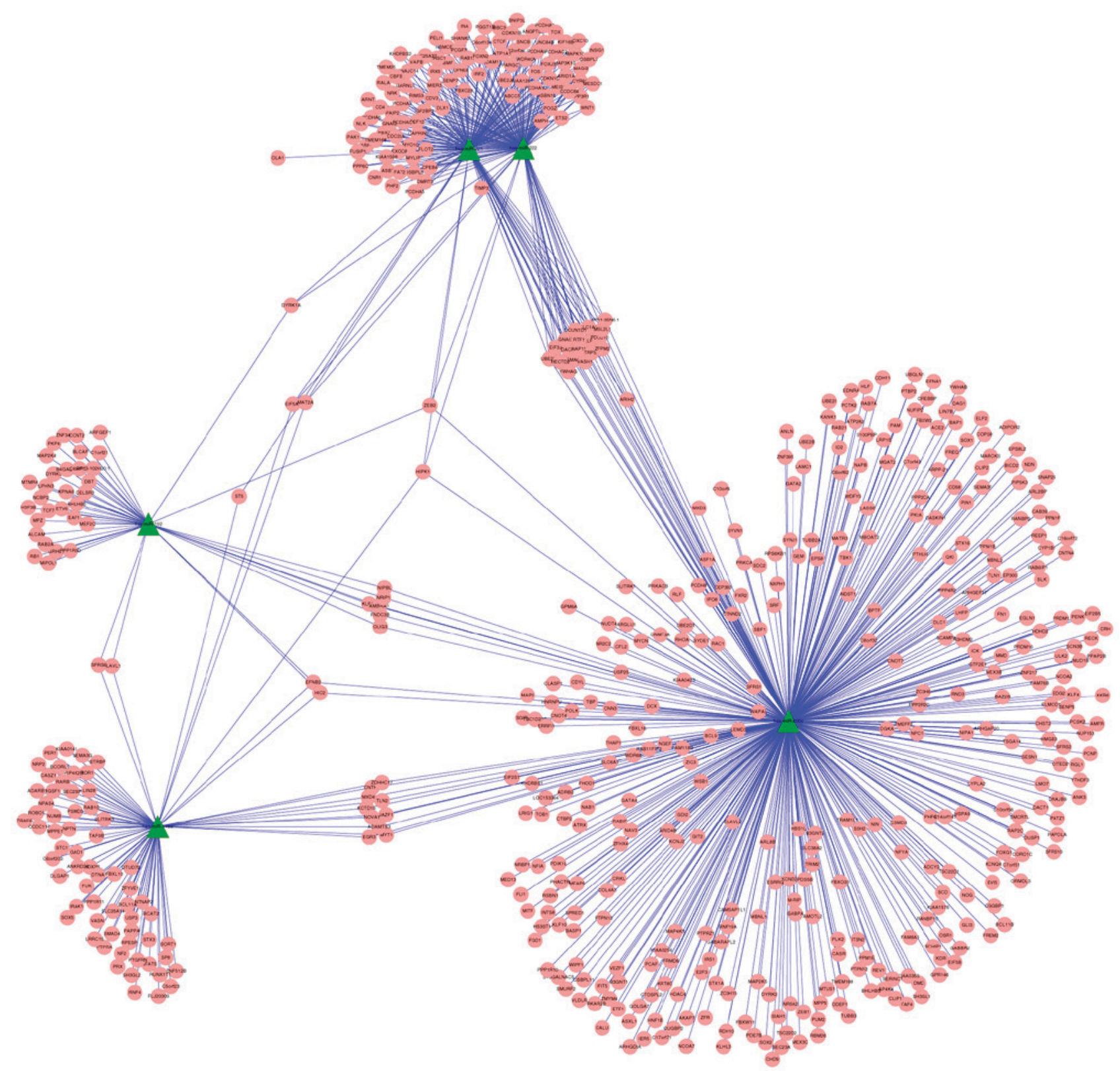

Figure 6. miRNA-gene network analysis constructed using Cytoscape software. Each cluster indicates an miRNA and its asscoaited genes. miRNA, microRNA.

responses. Although great improvements have been made in the treatment of breast cancer, such as endocrine therapy and HER2-targeted therapy, there are currently no available molecularly targeted therapeutics for patients with TNBC. Emerging evidence has demonstrated that miRNAs may serve as potential biomarkers and promising therapeutic targets for breast cancer $(13,14)$. In this study, miRNA microarray analysis of MDA-MB-231 cells (TNBC cells) and MCF-7 cells (breast adenocarcinoma luminal cells) was performed. It was reported by Terao et al that differential profiles of miRNA expression including miR-200c were determined in MCF-7 and MDA-MB-231 cells (15). In the present study, 107 miRNAs (57 upregulated and 50 downregulated) were identified to be differentially expressed in MDA-MB-231 cells compared with MCF-7 cells, suggesting that an altered expression of miRNAs may contribute to TNBC progression.
In this study, five prominently dysregulated miRNAs were further validated by RT-qPCR. The results were consistent with the results obtained by microarray analysis. It was demonstrated that miR-200c-3p expression levels in MDA-MB-231 cells were downregulated compared with MCF-7 cells. miRNA-gene network analysis also suggested that miR-200c shold be futher investigated for it's potential role in breast cancer. miR-200c has been demonstrated to serve important roles in the process of epithelial-to-mesenchymal transition by directly targeting the transcription factors zinc-finger E-box binding homeobox (ZEB)1 and ZEB2 $(16,17)$, two of the target genes in the analysis of the present study. miR-200c expression may induce a significant reorganization of tumor architecture, affecting important processes involved in cell adhesion and motility. Induced miR-200c expression significantly decreased the metastatic potential of a p53-knockout and low-claudin expressing tumor model with highly aggressive 
characteristics (18). miR-200c expression levels from TNBC patients was significantly reduced in cancer tissues compared with matched normal adjacent tissues by qPCR analysis (19). Overexpression of miR-200c decreased cell proliferation and promoted cell apoptosis in MDA-MB-231 cells by targeting the expression of X-linked inhibitor of apoptosis (19). It was reported that basal like breast tumor subtypes had lower levels of the miR-200 family compared with luminal subtypes (20), which was similar to the findings of the present study. In a previous study, it was found that the downregulation of miR-200c was associated with poor chemotherapy response in human breast cancer patients and the upregulation of miR-200c enhanced chemosensitivity to epirubicin partially via regulation of multi-drug resistance gene 1/P-glycoprotein expression (21). miR-200c was demonstrated to increase anoikis sensitivity by targeting nuclear factor- $\mathrm{\kappa B}$ and upregulated the tropomycin receptor kinase $\mathrm{B} /$ neutrophin 3 autocrine signaling loop in triple negative breast cancer (22). The miR-200 and miR-221 families were revealed to exert opposing effects on cellular plasticity during breast tumorigenesis (23). High miR-200 family expression promoted a well-differentiated epithelial phenotype whereas overexpression of miR-221/222 resulted in a poorly differentiated mesenchymal-like phenotype (23). A previous study demonstrated that knockdown of miR-221 inhibited cell migration and invasion by altering E-cadherin expression in TNBC cells (24). Søkilde et al (25) reported that there was an inverse association between miR-221/miR-222 and estrogen receptor expression by microarray analysis in luminal and basal breast cancer cell lines. A similar result was also found in highly invasive basal-like breast cancer cells and non-invasive luminal cells (26). It was demonstrated that miR-221/222 promoted S-phase entry and cellular migration in basal-like breast cancer (26). In this study, miR-221 and miR-222 expression levels were measured by RT-qPCR and were elevated in triple-negative type cells, which suggested that miR-221 and miR-222 may serve an important role in tumorigenesis in TNBC. In addition, it was found that miR-146a expression was significantly upregulated in MDA-MB-231 cells compared with MCF-7 cells. It was demonstrated that miR-146a downregulated BRCA1 expression, as confirmed by reporter assays (27). Another study reported that knockdown of miR-146a in BRCA1-overexpressing cells suppressed the ability to inhibit proliferation and transformation (28).

By using GO analysis, it was revealed that the target genes of aberrant miRNAs were associated with important processes such as regulation of metabolic processes, transcription factor activity and DNA binding. According to KEGG pathway enrichment analysis by using DAVID, the present study demonstrated that the most significant pathways included the MAPK signaling pathway, Wnt signaling pathway, TGF- $\beta$ signaling pathway, cAMP signaling pathway, AMPK signaling pathway, ubiquitin mediated proteolysis, cell cycle, adherens junction and gap junction, which were closely associated with tumor progression and metastasis. Increasing evidence has demonstrated that various and complex molecular signaling pathways were involved in TNBC progression and metastasis. The MAPK signaling pathway serves an important role in the regulation of cell proliferation, and aberrant activity of MAPK has been implicated in the development and progression of TNBCs (29). In a study involving 75 cases of TNBC patients with lymph-node metastases, it was demonstrated that phosphorylated extracellular-signal regulated kinase (pERK) expression was associated with longer event-free survival (duration from surgery until recurrence or mortality) and overall survival (duration from surgery until the date of mortality or the most recent date of follow-up) in TNBC, and $\mathrm{pERK}$ expression was an independent prognostic factor by multivariate analysis (30). Based upon gene expression profiles, diverse TNBC subtypes including basal-like 1 and basal-like 2 , immunomodulatory, mesenchymal, mesenchymal stem-like and luminal androgen receptor were identified (31). The mesenchymal and mesenchymal stem-like subtypes were enriched in signaling pathways involving TGF- $\beta$, mechanistic target of rapamycin, Wnt/ $\beta$-catenin, platelet-derived growth factor receptor, and vascular endothelial growth factor (31). It was found that treatment with Wnt pathway inhibitors in TNBC resulted in increased apoptosis and decreased cell proliferation and migration, which suggested that targeting the Wnt pathway may have therapeutic benefit for TNBC patients (32). It was reported that upregulation and enrichment of TGF- $\beta$ target genes were most frequent in mesenchymal stem-like TNBC or TNBC with low expression of claudin (33). TGF- $\beta$ signaling resulted in increased cell proliferation, migration, invasion and motility, whereas these effects were abrogated by a specific inhibitor against TGF- $\beta$ receptor I and the anti-diabetic agent metformin in breast cancer cell lines (33). In addition, a high expression of TGF- $\beta$ target genes were demonstrated to be associated with poor prognosis in claudin-low patients (33). The findings of the present study suggested that several signaling pathways are regulated by miRNAs including the MAPK pathway, Wnt pathway and TGF- $\beta$ pathway, and may serve significant roles in the development and progression of TNBC. Targeting these signaling pathways may have the potential to serve as a novel and promising strategy for the treatment of patients with TNBC.

In conclusion, 107 aberrant miRNAs in TNBC cells were identified using miRNA microarray analysis, and 597 potential target genes of five prominently dysregulated miRNAs that were validated by RT-qPCR were selected by miRNA target prediction algorithms. In addition, bioinformatic analysis suggested that certain pathways such as the MAPK, Wnt and TGF- $\beta$ signaling pathways were primarily involved in tumorigenesis and metastasis in TNBC. miRNA-gene network analysis suggested that miR-200c may contribute to breast cancer development. Investigating and validating key miRNAs and associated signaling pathways in TNBC patients compared with non-TNBC patients may be warranted in future work. Taken together, these findings may provide a view of the function of dysregulated miRNAs in TNBC, suggesting that dysregulated miRNAs may serve as potential biomarkers and therapeutic targets in TNBC.

\section{Acknowledgements}

The present study was supported by the grants from the Natural Science Foundation of Zhejiang Province (grant no.LQ13H160016), the Medical Science and Technology Program of Zhejiang Province (grant nos. 2013KYA026 and 2015DTA004) to JQ Chen, the Foundation of Science and Technology Department of Zhejiang Province 
(grant no. 2013C33205), the Medical Science and Technology Program of Zhejiang Province (grant no. 2013KYB034) to ZH Chen and the National Nature Science Foundation of China (grant no. 81672597) to XJ Wang.

\section{References}

1. Perou CM, Sørlie T, Eisen MB, van de Rijn M, Jeffrey SS, Rees CA, Pollack JR, Ross DT, Johnsen H, Akslen LA, et al: Molecular portraits of human breast tumours. Nature 406: 747-752, 2000.

2. Dent R, Trudeau M, Pritchard KI, Hanna WM, Kahn HK, Sawka CA, Lickley LA, Rawlinson E, Sun P and Narod SA: Triple-negative breast cancer: Clinical features and patterns of recurrence. Clin Cancer Res 13: 4429-4434, 2007.

3. Arnedos M, Bihan C, Delaloge S and Andre F: Triple-negative breast cancer: Are we making headway at least. Ther Adv Med Oncol 4: 195-210, 2012

4. Lewis BP, Burge CB and Bartel DP: Conserved seed pairing, often flanked by adenosines, indicates that thousands of human genes are microRNA targets. Cell 120: 15-20, 2005.

5. Bartel DP: MicroRNAs: Genomics, biogenesis, mechanism, and function. Cell 116: 281-297, 2004.

6. Izumiya $\mathrm{M}$, Tsuchiya $\mathrm{N}$, Okamoto $\mathrm{K}$ and Nakagama $\mathrm{H}$ : Systematic exploration of cancer-associated microRNA through functional screening assays. Cancer Sci 102: 1615-1621, 2011.

7. GwakJM,Kim HJ,Kim EJ, Chung YR, Yun S, Seo AN,Lee HJ and Park SY: MicroRNA-9 is associated with epithelial-mesenchymal transition, breast cancer stem cell phenotype, and tumor progression in breast cancer. Breast Cancer Res Treat 147: 39-49, 2014.

8. Eades G, Wolfson B, Zhang Y, Li Q, Yao Y and Zhou Q. lincRNA-RoR and miR-145 regulate invasion in triple-negative breast cancer via targeting ARF6. Mol Cancer Res 13: 330-338, 2015.

9. Yan LX, Huang XF, Shao Q, Huang MY, Deng L, Wu QL, Zeng YX and Shao JY: MicroRNA miR-21 overexpression in human breast cancer is associated with advanced clinical stage, lymph node metastasis and patient poor prognosis. RNA 14: 2348-2360, 2008.

10. Dennis G Jr, Sherman BT, Hosack DA, Yang J, Gao W, Lane HC and Lempicki RA: DAVID: Database for annotation, visualization, and integrated discovery. Genome Biol 4: P3, 2003.

11. Zhang X, Peng Y, Jin Z, Huang W, Cheng Y, Liu Y, Feng X, Yang M, Huang Y, Zhao Z, et al: Integrated miRNA profiling and bioinformatics analyses reveal potential causative miRNAs in gastric adenocarcinoma. Oncotarget 6: 32878-32889, 2015.

12. Smoot ME, Ono K, Ruscheinski J, Wang PL and Ideker T: Cytoscape 2.8: New features for data integration and network visualization. Bioinformatics 27: 431-432, 2011.

13. Kleivi Sahlberg K, Bottai G, Naume B, Burwinkel B, Calin GA, Børresen-Dale AL and Santarpia L: A serum microRNA signature predicts tumor relapse and survival in triple-negative breast cancer patients. Clin Cancer Res 21: 1207-1214, 2015.

14. Humphries B, Wang Z, Oom AL, Fisher T, Tan D, Cui Y, Jiang Y and Yang C: MicroRNA-200b targets protein kinase $\mathrm{C} \alpha$ and suppresses triple-negative breast cancer metastasis. Carcinogenesis 35: 2254-2263, 2014.

15. Terao M, Fratelli M, Kurosaki M, Zanetti A, Guarnaccia V, Paroni G, Tsykin A, Lupi M, Gianni M, Goodall GJ and Garattini E: Induction of miR-21 by retinoic acid in estrogen receptor-positive breast carcinoma cells: Biological correlates and molecular targets. J Biol Chem 286: 4027-4042, 2011.

16. Korpal M,Lee ES, Hu G and Kang Y: The miR-200 family inhibits epithelial-mesenchymal transition and cancer cell migration by direct targeting of E-cadherin transcriptional repressors ZEB1 and ZEB2. J Biol Chem 283: 14910-14914, 2008.

17. Gregory PA, Bracken CP, Smith E, Bert AG, Wright JA, Roslan S, Morris M, Wyatt L, Farshid G and Lim YY: An autocrine TGF-beta/ZEB/miR-200 signaling network regulates establishment and maintenance of epithelial-mesenchymal transition. Mol Biol Cell 22: 1686-1698, 2011.
18. Knezevic J, Pfefferle AD, Petrovic I, Greene SB, Perou CM and Rosen JM: Expression of miR-200c in claudin-low breast cancer alters stem cell functionality, enhances chemosensitivity and reduces metastatic potential. Oncogene 34: 5997-6006, 2015.

19. Ren Y, Han X, Yu K, Sun S, Zhen L, Li Z and Wang S: microRNA-200c downregulates XIAP expression to suppress proliferation and promote apoptosis of triple-negative breast cancer cells. Mol Med Rep 10: 315-321, 2014

20. Castilla MÁ, Díaz-Martín J, Sarrió D, Romero-Pérez L, López-García MÁ, Vieites B, Biscuola M, Ramiro-Fuentes S, Isacke CM and Palacios J: MicroRNA-200 family modulation in distinct breast cancer phenotypes. PLoS One 7: e47709, 2012.

21. Chen J, Tian W, Cai H, He H and Deng Y: Down-regulation of microRNA-200c is associated with drug resistance in human breast cancer. Med Oncol 29: 2527-2534, 2012.

22. Howe EN, Cochrane DR, Cittelly DM and Richer JK: miR-200c targets a NF- $\kappa$ B up-regulated TrkB/NTF3 autocrine signaling loop to enhance anoikis sensitivity in triple negative breast cancer. PLoS One 7: e49987, 2012.

23. Howe EN, Cochrane DR and Richer JK: The miR-200 and miR-221/222 microRNA families: Opposing effects on epithelial identity. J Mammary Gland Biol Neoplasia 17: 65-77, 2012.

24. Nassirpour R, Mehta PP, Baxi SM and Yin MJ: miR-221 promotes tumorigenesis in human triple negative breast cancer cells. PLoS One 8: e62170, 2013.

25. Søkilde R, Kaczkowski B, Podolska A, Cirera S, Gorodkin J, Møller S and Litman T: Global microRNA analysis of the NCI-60 cancer cell panel. Mol Cancer Ther 10: 375-384, 2011.

26. Li Y, Liang C, Ma H, Zhao Q, Lu Y, Xiang Z, Li L, Qin J, Chen Y, Cho WC, et al: miR-221/222 promotes S-phase entry and cellular migration in control of basal-like breast cancer. Molecules 19: 7122-7137, 2014.

27. Garcia AI, Buisson M, Bertrand P, Rimokh R, Rouleau E, Lopez BS, Lidereau R, Mikaélian I and Mazoyer S: Down-regulation of BRCA1 expression by miR-146a and miR-146b-5p in triple negative sporadic breast cancers. EMBO Mol Med 3: 279-290, 2011.

28. Kumaraswamy E, Wendt KL, Augustine LA, Stecklein SR, Sibala EC, Li D, Gunewardena S and Jensen RA: BRCA1 regulation of epidermal growth factor receptor (EGFR) expression in human breast cancer cells involves microRNA-146a and is critical for its tumor suppressor function. Oncogene 34: 4333-4346, 2015.

29. Giltnane JM and Balko JM: Rationale for targeting the Ras/MAPK pathway in triple-negative breast cancer. Discov Med 17: 275-283, 2014.

30. Hashimoto K, Tsuda H, Koizumi F, Shimizu C, Yonemori K, Ando M, Kodaira M, Yunokawa M, Fujiwara Y and Tamura K: Activated PI3K/AKT and MAPK pathways are potential good prognostic markers in node-positive, triple-negative breast cancer. Ann Oncol 25: 1973-1979, 2014.

31. Lehmann BD, Bauer JA, Chen X, Sanders ME, Chakravarthy AB, Shyr Y and Pietenpol JA: Identification of human triple-negative breast cancer subtypes and preclinical models for selection of targeted therapies. J Clin Invest 121: 2750-2767, 2011.

32. Bilir B, Kucuk O and Moreno CS: Wnt signaling blockage inhibits cell proliferation and migration, and induces apoptosis in triple-negative breast cancer cells. J Transl Med 11: 280, 2013.

33. Wahdan-Alaswad R, Harrell JC, Fan Z, Edgerton SM, Liu B and Thor AD: Metformin attenuates transforming growth factor beta (TGF- $\beta$ ) mediated oncogenesis in mesenchymal stem-like/claudin-low triple negative breast cancer. Cell Cycle 15: 1046-1059, 2016.

This work is licensed under a Creative Commons Attribution-NonCommercial-NoDerivatives 4.0 International (CC BY-NC-ND 4.0) License. 